\title{
A STUDY OF ARTIFICIAL NEURAL NETWORKS HYPERPARAMETER TUNING FOR DATA DRIVEN DECISION SYSTEMS
}

\author{
Adrian-Nicolae BUTURACHE \\ Bucharest University of Economic Studies, Romania \\ ad.buturache@yahoo.ro \\ Stelian STANCU \\ Bucharest University of Economic Studies, Romania \\ stelian.stancu@csie.ase.ro
}

\begin{abstract}
Targeted marketing based on data mining techniques can help the companies to understand their current and future customers in their efforts to deliver them best products and services. Artificial Neural Networks as universal approximators have the capability to find patters in data for delivering benchmark results in various domains for various classification or regression problems. Considering the digital transformation, available data and available communication channels between suppliers and customers it is becoming easier to identify the right customers and more cost effective to target then only. Technology advance and competition creates the premises for both success and failure to be just a matter of tuning the models in the best way for the selected use case. Moreover, it is not only about the industry. It is about the Artificial Neural Networks understanding added to the subject matter experts knowledge and experience. Moreover, Artificial Neural Networks can be applied with success in many other areas rather than marketing. Usually the limitations are not given by Artificial Neural Networks intrinsic characteristics but by the use case itself. This paper may engage marketing analysts or enthusiasts in using Artificial Neural Networks in their real-world use cases. Covering a wide range of knowledge from Artificial Neural Networks fundamentals to hyperparameter fine tuning and model evaluation being a guide for marketing practitioners in deploying their own solutions for data driven marketing campaigns.
\end{abstract}

Keywords: Artificial Neural Networks, Hyperparameter Tuning, Data Mining, Data Driven Decision

JEL classification: Model Evaluation, Artificial Neural Networks, Customer Data

DOI: $10.24818 / \mathrm{ie} 2020.04 .03$

\section{Introduction}

Targeted marketing identifies a group of people who are likely to buy a service or product, and then the service or product is promoted only to the group previously identified for purchase. In this way the promotion messages are transmitted directly to the members of the identified groups with great potential to purchase, instead of being sent to a large mass of people who can buy or not. This allows the efforts to be concentrated towards groups for which the services or products to be promoted have relevance, leading to an increase in the rate of transformation of potential customers into actual customers. Nowadays mass marketing impact on customers is not meeting the expectations [1]. If 20 years ago marketing meant TV ads, magazines or newspapers, currently marketing campaigns are conducted by a large proportion in the online environment, an environment unavailable on such a scale in the past. Innovation in the field of information technology such as large-scale adaptation of high-speed internet, cheap internet 
services and mobile smart devices, the emergence of social networks, all have led through digital transformation [2], to the creation of new rules in marketing. Marketing facilitates the relationship between customers and suppliers by mapping customers behaviors, expectations and experiences, transmitting them to the suppliers who can shape their services and products accordingly. From the point of view of digital marketing development, so far, 3 important roles have been identified in 3 different periods of time, approximately 5 years each: facilitator of individual expression, decision support tool, marketing intelligence [3]. On the other hand, advertising messages unsolicited or unwanted by the potential customers represent what is generally known as spam [4]. This topic has been included in European law as part of the protection of electronic communication (2002/58/EC) and only e-mail messages were included. The multitude of communication channels available have created the premises to reinforce the law across EU and to add the EU General Data Protection Regulation (GDPR) in 2018. Going further and understanding the impact of the new technologies on the way marketing is made a new type of communication was been developed, two-way communication between customers and suppliers, both groups sharing same virtual space [5]. Through social media companies can share with their customers and future customers content about products driving in a new way marketing initiatives. Moving to Big Data conventional methods can start to lose the capability of making the right prediction. Moreover, taking advantage of the new data that is now available it is allowing to perform deep dives for data insights that were never revealed before Big Data. RFM is covering 3 dimensions in customer analysis: Regency, Frequency and Monetary Value. Basically, this method is analyzing how recently a customer completed a purchase, how often is purchasing and how much is the amount of money spent [6].

Artificial Neural Networks can be used to solve one of the most commune targeted marketing use case, classification of potential customers as buyers or non-buyers. Multilayer perceptron can be deployed with very good results in direct marketing and fraud detection [7]. Moreover, the algorithm can be customized to be profit-driven by selecting an objective function that is maximizing the profit. This is possible by associating a different weight to each instance based on the importance. In this way the misclassification of each instance is impacting in a different way the result. The difference between this approach and the approach in which each class is having same importance is not big but, in some cases, the first one is performing slightly better. Direct-marketing campaigns represents one of the marketing pillars [8]. For a model aiming to provide optimum way to select the right clients to receive one or multiple offers during a marketing campaign the studied problem can become strongly NP-hard and finding the optimum based on a constant-factor approximation algorithm can become impossible. Multiple heuristics were developed based on integer programming. Conducting activities associated with direct-marketing in the right way can generate profit in two ways: customer acquisition, when new customers are targeted and transformed from potential customers into actual customers and customer retention, when existing customers are kept by selling them new services or products based on their customer profile [9]. Artificial Neural Networks can be used to map and model the relationship between customer and supplier based on the attributes that are creating and increasing loyalty [10]. Indonesian coffee market growth it is generated by the Indonesian customers which started to include drinking coffee in their daily lifestyle. A Multilayer perceptron is used to classify the customers based on their level of loyalty, 1, for higher preference, and 0 , for lower preference. A high preference is considered when customers have consumed repeatedly same instant coffee brands. The conclusion based on the research is that the most influencing variables in this analysis are related to the product appearance like size, package, design, product information on the package, coffee differentiation. Proposed ANN predicted well the customers preferences in $58.2 \%$ of the cases. An interesting use case 
is represented by the marketing strategy built based on Artificial Neural Networks, Decision trees and Support Vector Machine [11]. The aim is to identify locations for educational events organized for healthcare professionals using data analytics. Support Vector Machines and Artificial Neural Network models performed better than Decision trees in classifying if an event should be scheduled or not in a certain location. Another dimension in the relationship between suppliers and customers is that one related to the emotional responses generated by the marketing campaigns [12]. Multiple Feedforward Neural Networks are used to model data gathered from the customers through surveys. The results are meaningful for the two companies involved in the study giving insights about both slogans used in marketing campaigns and customers emotional responses. Same data set used in this research papar has been analyzed using Artificial Neural Networks, Decision Trees, Support Vector Machine, Logistic Regression [13] and a hybrid approach called Neuro-fuzzy approach. The Neuro-fuzzy systems are used to substitute the lack of transparency of the Artificial Neural Networks considered to be black boxes. The mathematics and intrinsic fuzzy rules represent the domain knowledge [14]. In 1957 Frank Rosenblatt presented an improved version of McCulloch and Pitts work called perceptron. By modifying the absolute inhibition and unit equal synaptic weights constrains his model was actually able to learn from data. The way model was learning was called further supervised learning. The values of the synaptic weights were adjusted based on the training data feed into the model. Another adding on the first proposed model it is the bias, a constant input value with a similar role as a synaptic weight.

\section{Artificial Neural Networks}

In biology a neuron has the main function to process information. In the brain neurons are building blocks which are communicating with other cells using special connections. The dendrites receive signals (impulses) from other neurons and are send them to the cell body where the information is processed. Signals generated by the cell body are transmitted further along axon which it is branching into strands. The region between an axon of a neuron and a dendrite of another one is called synapse. This functional unit releases certain chemicals (neurotransmitters) based on the electrical impulse received from the cell body via axon. Based on the nature of the receptor neuron those neurotransmitters can enhance or inhibit further electrical impulses. The synapse behavior can be changed in time based on the signals passing through it. This process and its explanation can be transposed into what we are calling learning in both biology and Artificial Neural Network theories.

Warren McCulloch and Walter Pitts published in 1943 for the first time a paper where they explained their concept about neural networks [15].

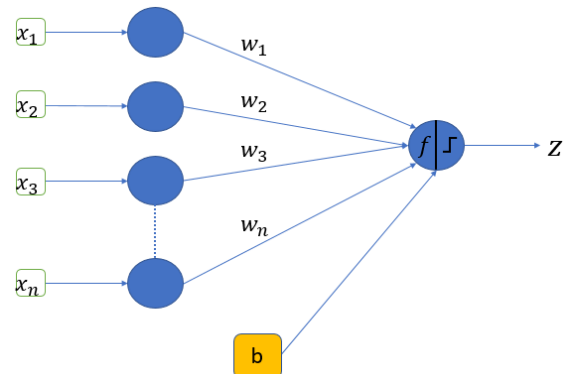

Figure 1. Mathematical representation of the neuron model proposed by Rosenblatt

Each neuron is having its own input $\left(x_{1}, x_{2}, x_{3}, \ldots, u_{n}\right) \in R^{n}$. Transformation $f$ is applied on the weighted sum of the input variables. The output of a neuron can be calculated as following:

$$
y=f(z)
$$




$$
z=\sum_{i=1}^{n} u_{i} w_{i}+b
$$

Where $\mathrm{b}$ is the bias and $w=\left(w_{1}, w_{2}, \ldots, w_{m}\right) \in R^{m}$ represents the weights vector.

Most simple calculation for the output:

$$
y=f(z)= \begin{cases}1, & \text { if } w u+b \geq \tau \\ 0, & \text { if } w u+b<\tau\end{cases}
$$

Where $\tau$ represents the threshold.

Artificial Neural Networks are learning by adjusting the synaptic weights during the training process. A major role is represented by the loss function which is saying how close is the prediction to the actual values. This function can be named cost function or error function as well. Loss function selection is a matter of studied problem. For classification cross-entropy is preferred. A maximum likelihood loss function as Binary Cross-Entropy is making an estimation of the closeness between predicted values and actual values. Therefore, the final objective of the network is to provide answers (predictions) as close as possible to the actual values.

$$
\text { Loss }=-(y \log (p)-(1-y) \log (1-p))
$$

Where here $y$ is 1 or 0 if the class label is correct or not correct classified and $\mathrm{p}$ is the predicted probability of an observation to be part of the class in which was predicted.

Cross-entropy is used to measure the error between two probability distributions under maximum likelihood. Aim is to find best synaptic weights combination to minimize the difference between predicted and actual probability distributions.

\section{Hyperparameters}

Batch size can be defined as the numbers of samples that are propagated through the neural network. Gradient Descent it is a first order optimization algorithm considering first derivative only when parameters update is made. Synaptic weights are updated to minimize the model error over the training set. Neural network parameters are update in the opposite direction of the objective function's gradient. Learning rate is determining the size of the step on each iteration.

Over the last years due to the increase of data available for analysis an increase of the research activities for finding optimization algorithms scalable based on the use case [16]. Stochastic gradient descent become one of the popular choices of the practitioners. Gradient descent is one of optimization algorithms used for synaptic weights calculation and update. Let's consider a function $y=f(x), y$ and $x$ both real numbers. The derivative of $f(x), f^{\prime}(x)$ or $\frac{d y}{d x}$, gives the slope of it in a certain point $x$ [17]. The derivative provide information about how to change $x$ to minimize $y$. Reducing $f(x)$ by moving $x$ in the opposite of the derivative is called gradient descent. The point in which derivatives does not provide anymore the direction where to move the value $x$ is called critical point, $f^{\prime}(x)=0$. For use cases with multiple inputs partial derivatives $\frac{d f}{d x_{i}}$ will measure how $x_{i}$ is impacting the function $f$ in a certain $x$ points. $\nabla_{x} f(x)$ represents the vector of all partial derivatives. Extending the logic of the above-mentioned critical point in case of multiple dimensions for each gradient element must be 0 . Using the intuition explained above Gradient descent method for finding a new point is the following: 


$$
\omega_{t+1}=\omega_{t}-\eta \nabla_{\omega} J(\omega)
$$

where $\eta$ represent the learning rate $\in(0,1), J(\omega)$ represent objective function and model parameters are represented by $\omega, \omega_{t+1}$ is the future state of the model parameters and $\omega_{t}$ is the current state.

In the Batch Gradient Descent, the error is calculated, as for the Stochastic Gradient Descent, for each example of the training set but the model parameters are updated after all training examples are passing through the network. One passing through the network is called epoch. Therefor model parameters are updated after an epoch is completed. This approach can decrease the computational power and training time and could enable parallel processing since error calculation and model parameters update are separated. Over large batches the advantage of faster training could disappear. Batch size is equal to the size of the training set. Batch Gradient Descent method update equation:

$$
\omega_{t+1}=\omega_{t}-\eta \nabla_{\omega} J\left(\omega ; x^{(i)} ; y^{(i)}\right)
$$

Stochastic Gradient Descent represents an instance of the Gradient Descent algorithm that calculates error and the synaptic weights updates after each example of the training set. This approach could insure for some use cases faster learning, better generalization capability by avoiding local minimum. On the other hand, same upsides can lead to slow convergence, longer training time and costly computation since the model is updated for each training set example. Batch size is equal to 1 .

Mini-Batch Gradient descent represents a solution that is aiming to deploy in the same time Batch Gradient Descent and Stochastic Gradient Descent. Small batches of training data are feed into the neural network, over each mini-batch the mean gradient is calculated and further the synaptic weights are updated. Batch size is equal to a value bigger than 1 and smaller than training data set size. Mini-Batch Gradient Descent method update equation:

$$
\omega_{t+1}=\omega_{t}-\eta \nabla_{\omega} J\left(\omega ; x^{(i i i+n)} ; y^{(i i i+n)}\right)
$$

Parameters are updated after every mini-batch containing $n$ training examples is passed through the network.

Adagrad (Adaptive Gradient Algorithm) is part of the Adaptive stochastic gradient descent methods. It is known and demonstrated that can perform well for non-convex stochastic optimization [18]. Adagrad algorithm is adapting all learning rates of the model parameters. This is possible by scaling the learning rates based on the partial derivative of the loss, the greater the derivative the faster the decrease of the associated learning rate. For the parameters having small partial derivative of the gradient the decrease of the associated learning rate is also smaller [19].

$$
\begin{gathered}
\omega_{t+1}=\omega_{t}-\frac{\eta}{\sqrt{G_{t}+\varepsilon}} \nabla_{\omega} J(\omega) \\
G_{t}=G_{t-1}+\left[\nabla_{\omega} J(\omega)\right]^{2}
\end{gathered}
$$

And where $\varepsilon$ is a constant included to avoid possible scenarios in which the division would have been possible to be made by zero. Based on (8) learning rate is divided by the square root of the sum of past and current squared gradients. Due to the way that the squared gradients are used iteration by iteration the cumulative value of the denominator is growing as the number of iterations is increasing. The consequence is that the learning rates are becoming smaller and smaller until the point where the algorithm is no longer acquiring knowledge.

RMSProp or Root Mean Square Prop as Adagrad is an adaptive learning rate optimization algorithm [20]. This solution was proposed to solve Adagrad's shortcoming on diminishing 
learning rates. Taking the exponential moving average of the gradients instead of cumulative sum of squared gradients it will overcome this weakness of the Adagrad algorithm. The update equation remains the same as in (8) the difference is made on the denominator term $G_{t}$.

$$
G_{t}=\beta G_{t-1}+(1-\beta)\left[\nabla_{\omega} J(\omega)\right]^{2}
$$

Adam is a method for stochastic optimization based on adaptive estimates of low-order moments [21]. The name of this method is come from adaptive moment estimation. Is combining the advantages of Adagrad and RMSProp working well for sparse gradients, under on-line and non-stationary framework, being computationally and memory efficient. Adam is using exponential moving average of gradient $\left(m_{t}\right.$ - the estimate of the first moment (mean) of the gradient) for scaling the learning rate instead of the simple average used in Adagrad and squared gradient ( $v_{t}$ the second raw moment (the uncentered variance) of the gradient) as in RMSProp.

$$
\begin{gathered}
m_{t}=\beta_{1} m_{t-1}+\left(1-\beta_{1}\right) \nabla_{\omega} J(\omega) \\
v_{t}=\beta_{2} v_{t-1}+\left(1-\beta_{2}\right)\left[\nabla_{\omega} J(\omega)\right]^{2}
\end{gathered}
$$

$\beta_{1}$ and $\beta_{2}$ represents hyper-parameters $\in[0,1)$ used for controlling the exponential decay rates of the moving averages $\left(m_{t}\right)$ and $\left(v_{t}\right)$.

During the initial time steps due to the initialization as vectors of 0 and for values of $\beta_{1}$ and $\beta_{2}$ are close to 1 then $m_{t}$ and $v_{t}$ are biased towards 0 . To avoid this drawback bias-corrected estimates of $m_{t}$ and $v_{t}$ are proposed

$$
\begin{aligned}
& \hat{m}_{t}=\frac{m_{t}}{1-\beta_{1}^{t}} \\
& \hat{v}_{t}=\frac{\hat{v}_{t}}{1-\beta_{2}^{t}}
\end{aligned}
$$

And in this case the update method is the following:

$$
\omega_{t+1}=\omega_{t}-\frac{\eta}{\sqrt{\hat{v}_{t}+\varepsilon}} \hat{m}_{t}
$$

Adamax is an evolution of the Adam optimizer deploying a p-norm based update rule. For the use cases in which $\mathrm{p} \rightarrow \infty$ then the algorithm become simple and stable.

Update steps for Adamax:

$$
\begin{gathered}
m_{t}=\beta_{1} m_{t-1}+\left(1-\beta_{1}\right) \nabla_{\omega} J(\omega) \\
u_{t}=\max \left(\beta_{2} u_{t-1},\left|\nabla_{\omega} J(\omega)\right|\right)
\end{gathered}
$$

Where $u_{t}$ represents the update of the exponentially weighted infinity norm.

Finally, the update for all network parameters:

$$
\omega_{t}=\omega_{t-1}-\frac{\eta}{\left(1-\beta_{t}^{1}\right)} \frac{m_{t}}{u_{t}}
$$

Weights initialization is paying an important role in the overall network training. A proper initialization proved to be critical in having a network that is converging to the desired minima in a reasonable amount of time or in the opposite scenario the training could spread on bigger time frame with even less performance. For an initialization with a constant value all neurons will have an identical impact on the cost which leading to identical gradients. Further during 
the training, neurons will evolve symmetrically, restricting neurons in acquiring different knowledge. Moreover, having initial values too big or too small will lead to enlarging or vanishing gradients. Both cases generating poor performance of the network. Randomly selecting small numbers out of a normal distribution will drive to a variance of the weights as function of the number of inputs. The initialization strategies deployed were chosen to overcome the weaknesses presented above. LeCun initialization methodology suppose drawing the initial synaptic weights values from a distribution with zero mean and a certain variance [22]. Two instances of this methodology are widely deployed, both solving the dependence of variance on number of inputs. LeCun Normal Initialization is taking a truncated normal distribution centered in zero and with a standard deviation $\sigma_{\omega}$ described by:

$$
\sigma_{\omega}=\sqrt{\frac{1}{\text { input_dim }}}
$$

Where input_dim is the number of connections feeding into the neuron. LeCun Uniform Initialization is drawing the samples out of a distribution bounded lower and superior:

$$
\begin{aligned}
& \text { lower bound }=-\sqrt{\frac{3}{\text { input_dim }}} \\
& \text { upper bound }=\sqrt{\frac{3}{\text { input_dim }}}
\end{aligned}
$$

Xavier initialization is an evolution of LeCun's work but instead of taking into consideration only the number of input units in the weight tensor is taking also the number of output units in the weights tensor [23]. Xavier Normal Initializer is using samples drawed from a truncated normal distribution centered on 0 with the standard deviation $\sigma_{w}$ :

$$
\sigma_{w}=\sqrt{\frac{2}{\text { input_dim+output_dim }}}
$$

Where input_dim is the number of inputs units in the weight tensor and output_dim is the number of output units in the weight tensor. Xavier Uniform Initializer is drawing the samples from a uniform distribution bounded lower and upper.

$$
\begin{aligned}
& \text { lower bound }=-\sqrt{\frac{6}{\text { input_dim }+ \text { output_dim }}} \\
& \text { upperbound }=\sqrt{\frac{6}{\text { input_dim+output_dim }}}
\end{aligned}
$$

Activation functions are triggering neuron to transfer or to not transfer the signal further. In literature activation functions are known also as transfer functions. Activation functions can be divided in two categories: linear activation functions and non-linear activation functions. Without activation functions the neural network model is just a linear regression model. Linear activation function is one of the simplest solutions:

$$
y_{i}=m z_{i}=f\left(z_{i}\right)
$$

The derivative of the function with respect to $z_{i}$ is $m$. This means there is no relation to the input $z_{i}$. 
Sigmoid or logistic activation function is one of the most used in feedforward neural networks due to some of its advantages like being nonlinear and computational simplicity of its derivative [24]. It is continuously differentiable function satisfying the relation:

$$
y_{i}=\frac{1}{1+\exp \left(-z_{i}\right)}=f\left(z_{i}\right)
$$

The output of the sigmoid activation function is always bounded in the range $(0,1)$. Most important pitfall while using the sigmoid functions are the extreme ends, almost horizontal regions in which where the responses on the changes in $z$ are diminished. It is used for the models where the output is represented by a probability, like classification problems. A more general logistic activation function is Softmax and is used for multiclass classification.

Tanh is an activation function similar with sigmoid being a scaled sigmoid function, existing in the range $(-1,1)$.

$$
\begin{gathered}
\tanh \left(z_{i}\right)=2 \operatorname{sigmoid}\left(z_{i}\right)-1 \\
f\left(z_{i}\right)=\tanh \left(z_{i}\right)=\frac{2}{1+\exp \left(-2 z_{i}\right)}
\end{gathered}
$$

Tanh as sigmoid is exposed as well on diminished responses for the close to horizontal regions. Relu or Rectified linear unit is one of the most popular activation functions among deep learning practitioners. Relu is more computationally efficient than sigmoid and tanh activation functions by activating only some of the neurons at same time.

$$
f\left(z_{i}\right)=\max \left(\left(0, z_{i}\right)\right.
$$

On the other hand, this can become a weakness since could lead to a situation in which a large percentage of the neurons will not be activated anymore during the training. Being less expensive than sigmoid and tanh in terms of computation is enabling a faster convergence [26]. An Artificial Neural Network architecture should provide a robust solution for studied use case. Robustness can be evaluated through the network performance. An architecture that is providing minimal error indicate that the network can generalize [26]. The number of neurons on the hidden layer can be fixed using existing knowledge based on trials or can be adapted based on the use case [27]. One approach for the number of neurons on the hidden layer of an MLP is specified by Kolmogorov, 1957. In this theory the number of neurons on the hidden layers should be $2 n+1$, where $n$ is the number of neurons of the input vector.

When the number of hidden layers is analyzed, and the exact number of layers must be chosen it is important to know the type of problem that the ANN will deal. For applications where, linear separable functions need to be represented, a single layer perceptron can be enough to make a split. An example of application can be in this case the binary classification. For highdimensional use cases where linear separability is not enough to split certain classes, where separability plane is needed MLP is used.

Underfitting and overfitting can be explained also by analyzing the number of neurons inside hidden layers [28]. When the number of neurons is not big enough to capture the complexity of the relations between variables inside the data set under fitting may appear. On the other hand, when the number of neurons inside hidden layers is bigger than the number of neurons needed to map the relations between variables this might generate over fitting.

\section{Methodology}

CRISP-DM (Cross-Industry Standard for Data Mining) is a standard that is defining step by step data mining process. SAS institute proposed before CRISP-DM their own data mining framework called SEMMA (Sample, Explore, Modify, Model, Assess). In 1996, SPSS, 
Teradata, Daimler AG, NCR and OHRA joined their forces to build what the data mining standard that become nowadays Cross Industry Standard for Data Mining. In 2015 IBM published its own instance of CRISP-DM. A refined standard called ASUM-DM (Analytics Solutions Unified Method for Data Mining/ Predictive Analytics). CRISP-DM is a living document being updated and refined multiple times in the last 20 years and based on the dynamics inside analytics ecosystem it looks like CRISP-DM will continue to evolve.

The main phases of CRISP-DM framework are the following: Business Understanding, Data understanding, Data Preparation, Modeling, Evaluation and Deployment. For this paper just the first five phases will be followed, deploying the model being out of scope. The framework can be customized and shaped on the user's goals and resources.

\section{Data overview}

The data set used in this analysis was generated during a marketing campaign based on phone calls made available by University of California at Irvine (UCI). S. Moro, R. Laureano and P. Cortez collected the data and built the data set. Moreover, they built their own predictive analysis and their results will be the base line for this analysis [29]. Used data set contains 41188 records, 20 independent variables and 1 dependent variable. Data set variables are split in numerical (Age, Duration, Campaign, Pdays, Previous, Employment variation rate, Consumer price index, Consumer confidence index, Euribor 3-month rate, Number of employees) and categorical (Job, Marital, Education, Default, Housing, Loan, Contact, Month, Day of week, Poutcome, Output).

Taking into consideration the learning scenario and based on the data available this research is falling in the supervised learning category. Supervised learning is a paradigm in which the algorithm is feed with labeled data as training data and must make predictions for unseen data. This means the algorithm will need to identify patterns inside the training data that will allow it to predict the correct label of the unseen data [30]. To be more specific for this analysis a binary classification is requested.

Before feeding the data into the neural network one crucial step is to transform the categorical data using one-hot encoding [31] since neural networks are not capable deal with categorical variables. New features are built for day of week and month using the date variable. At the end of this step 19 out of 81 independent variables are removed due to multicollinearity. Data set split between training and testing is $80 \%$ for training and $20 \%$ for testing during the entire study.

\section{Evaluation}

To find the best architecture multiple optimization scenarios are performed. First step is to select the metrics and to set the baseline. Once the baseline is set the optimization can start. In terms of performance understanding Confusion matrix represents the starting point when a classification model performance is assessed. Confusion matrix it is a 2D "Actual" and "Predicted" table. Inside confusion matrix are the following values: True Positives (TP) Actual class of the point to be predicted as True (1), True Negatives (TN) - Actual class of the point to be predicted was False (0), False Positive (FP) - Actual class of the point to be predicted was True (1) but the prediction was False (0), False Negative (FN) - Actual class of the point to be predicted was False (0) but the prediction was True (1) [32].

In an ideal case it is desirable to minimize both False Positive and False Negative. Based on use case in real world problems it is needed to minimize False Positive Rate or False Negative Rate. False Positive Rate (FPR) or Type I error represents represent the fraction of alerts when a point was predicted to be true put the actual value was false. 


$$
F P R=\frac{F P}{F P+F N}
$$

False Negative Rate or Type II error represents fraction of the data points for which we missed to predict the False (0).

$$
F N R=\frac{F N}{T P+F N}
$$

For highly imbalanced data sets FPR or FNR tend to be low or high based on which class of the data set is imbalanced.

For this use case Type II errors count is higher than Type I errors because the TN class is bigger than TP class and Type II errors are calculated using TN as part of the denominator.

Specificity or True Negative Rate measures how well the False (0) observations were classified as False (0).

$$
T N R=\frac{T N}{T N+F P}
$$

True Positive Rate or Recall or Sensitivity represents it's a measure of observations classified as positive out of all possible positive observations

$$
F P R=\frac{T P}{T P+F N}
$$

Positive Predictive Value or Precision represents how well the True (1) points are predicted as True (1).

$$
P P V=\frac{T P}{T P+F P}
$$

Accuracy is a measure of both True and Positive points classified correctly

$$
A C C=\frac{T P+T N}{T P+F P+T N+F N}
$$

Overcoming this issue F1 Score combines the Recall and Precision into a single Metric by making the harmonic bean between these two.

$$
F_{1}=2 \frac{\text { Sensitivit } \cdot \text { Precision }}{\text { Sensitivit }+ \text { Precision }}
$$

AUC (Area Under the Curve) - ROC (Receiver Operating Characteristics) curve is a measure of how good is the model in distinguishing classes based on predicted probability. False Positive Rate is on the $\mathrm{X}$ - axis and True Positive Rate is on the $\mathrm{Y}$-axis in most typical approach. Higher the AUC, better the model is making the predictions, distinguishing between classes. Considering the multitude of metrics available for this type of classification and the use case in this paper AUC it is selected for measuring the performance of the tested algorithms while F1 Score and Accuracy are used to decide for closer AUC values which combination of hyperparamenters it is performing better. There is no rule on how many combinations of hyperparameters will continue after every optimization, it is a matter of how promising the results are.

\section{Results}

The neural network deployed initially to set the baseline is a Multi-layer Perceptron with 61 input variables, Xavier uniform weights initialization and Adam optimizer. Hidden layer consists of 123 neurons ( $2 n+1$, where $n$ is the number of input variables) with Relu activation 
function. Output layer is represented by a single neuron with Sigmoid activation function. Baseline metrics for are: $\mathrm{AUC}=0.9377, \mathrm{~F} 1 \mathrm{Score}=0.5174$, Accuracy=0.9124.

Table 1. Epochs and Batch optimization

\begin{tabular}{|l|l|l|l|l|l|l|l|l|l|l|l|}
\hline & $\mathbf{e 1}$ & $\mathbf{e 1 0}$ & $\mathbf{e 2 0}$ & $\mathbf{e 3 0}$ & $\mathbf{e 4 0}$ & $\mathbf{e 5 0}$ & $\mathbf{e 6 0}$ & $\mathbf{e 7 0}$ & $\mathbf{e 8 0}$ & $\mathbf{e 9 0}$ & $\mathbf{e 1 0 0}$ \\
\hline $\begin{array}{l}\text { AUC } \\
\text { b10 }\end{array}$ & 0.9406 & 0.9414 & 0.9351 & 0.9312 & 0.9238 & 0.9228 & 0.9175 & 0.9120 & 0.9168 & 0.9161 & 0.9138 \\
\hline $\begin{array}{l}\text { AUC } \\
\text { b30 }\end{array}$ & 0.9408 & 0.9419 & 0.9371 & 0.9348 & 0.9304 & 0.9275 & 0.9199 & 0.9197 & 0.9202 & 0.9151 & 0.9142 \\
\hline $\begin{array}{l}\text { AUC } \\
\text { b50 }\end{array}$ & 0.9387 & 0.9436 & 0.9393 & 0.9377 & 0.9332 & 0.9306 & 0.9251 & 0.9247 & 0.9204 & 0.9205 & 0.9154 \\
\hline $\begin{array}{l}\text { F1 Score } \\
\text { b10 }\end{array}$ & 0.5915 & 0.5770 & 0.5571 & 0.5867 & 0.5479 & 0.5420 & 0.5503 & 0.5295 & 0.5570 & 0.5338 & 0.5506 \\
\hline $\begin{array}{l}\text { F1 Score } \\
\text { b30 }\end{array}$ & 0.5637 & 0.5627 & 0.5468 & 0.5807 & 0.5432 & 0.5405 & 0.5570 & 0.5454 & 0.5804 & 0.5494 & 0.5540 \\
\hline $\begin{array}{l}\text { F1 Score } \\
\text { b50 }\end{array}$ & 0.5525 & 0.5798 & 0.5649 & 0.5960 & 0.5515 & 0.5450 & 0.5636 & 0.5701 & 0.5617 & 0.5511 & 0.5497 \\
\hline $\begin{array}{l}\text { Accuracy } \\
\text { b10 }\end{array}$ & 0.9182 & 0.9153 & 0.9120 & 0.9101 & 0.9054 & 0.9034 & 0.8980 & 0.8965 & 0.8996 & 0.8961 & 0.8997 \\
\hline $\begin{array}{l}\text { Accuracy } \\
\text { b30 }\end{array}$ & 0.9156 & 0.9149 & 0.9119 & 0.9108 & 0.9096 & 0.9104 & 0.8996 & 0.9008 & 0.9047 & 0.8999 & 0.8982 \\
\hline $\begin{array}{l}\text { Accuracy } \\
\text { b50 }\end{array}$ & 0.9156 & 0.9178 & 0.9142 & 0.9134 & 0.9096 & 0.9104 & 0.9013 & 0.9081 & 0.9017 & 0.9051 & 0.8988 \\
\hline
\end{tabular}

In the Table 1. where b stands for batch and e stand for epochs biggest AUC value is obtained after training the initial architecture over 10 epochs and using mini-batches of 50. Bigger the number of epochs, lower the AUC. Regarding the mini-batch size, nu significant improvement appears for values bigger than 50 .

Table 2. Optimization algorithm optimization

\begin{tabular}{|c|c|c|c|c|c|c||c|}
\multicolumn{1}{c|}{} & \multicolumn{1}{c|}{ Table 2. Optimization algorithm optimization } \\
\multicolumn{1}{c|}{$\begin{array}{c}\text { Adagrad } \\
\text { b10 e10 }\end{array}$} & $\begin{array}{c}\text { Adagrad } \\
\text { b30 e10 }\end{array}$ & $\begin{array}{c}\text { Adagrad } \\
\text { b50 e10 }\end{array}$ & $\begin{array}{c}\text { Adam } \\
\text { b10 e10 }\end{array}$ & $\begin{array}{c}\text { Adam } \\
\text { b30 e10 }\end{array}$ & $\begin{array}{c}\text { Adam } \\
\text { b50 e10 }\end{array}$ & $\begin{array}{c}\text { Adamax } \\
\text { b10 e10 }\end{array}$ \\
\hline AUC & 0.9456 & 0.9455 & 0.9450 & 0.9416 & 0.9424 & 0.9426 & 0.9430 \\
\hline F1 Score & 0.5978 & 0.5854 & 0.5947 & 0.5857 & 0.5696 & 0.5803 & 0.5667 \\
\hline Accuracy & 0.9206 & 0.9187 & 0.9198 & 0.9155 & 0.9171 & 0.9166 & 0.9144 \\
\hline & $\begin{array}{c}\text { Adamax } \\
\text { b30 e10 }\end{array}$ & $\begin{array}{c}\text { Adamax } \\
\text { b50 e10 }\end{array}$ & $\begin{array}{c}\text { RMSProp } \\
\text { b10 e10 }\end{array}$ & $\begin{array}{c}\text { RMSProp } \\
\text { b30 e10 }\end{array}$ & $\begin{array}{c}\text { RMSProp } \\
\text { b50 e10 }\end{array}$ & $\begin{array}{c}\text { SGD b1 } \\
\text { e10 }\end{array}$ & \\
\hline AUC & 0.9439 & 0.9445 & 0.9358 & 0.9403 & 0.9414 & 0.9428 & \\
\hline F1 Score & 0.5757 & 0.5840 & 0.5831 & 0.5672 & 0.5769 & 0.5860 & \\
\hline Accuracy & 0.9181 & 0.9185 & 0.9193 & 0.9155 & 0.9161 & 0.9170 \\
\hline
\end{tabular}

Adagrad and Adamax are performing better than Adam. Based on the theoretical aspects of these 3 optimizers highlighted above the expectation was to have both Adam and Adamax performing better than Adagrad. Additionally, the impact of the mini-batch size is limited for Adagrad and Adamax. Moreover, SGD or Stochastic Gradient Descent it is performing better than Adam and RMSProp event the last two are taking the advantage of adaptive learning.

Table 3. Learning Rate Optimization 
Proceedings of the IE 2020 International Conference

www.conferenceie.ase.ro

\begin{tabular}{|c|c|c|c|c|c|c|c|c|}
\hline & $\begin{array}{c}\text { Adamax } \\
\text { b50 e10 } \\
\text { lr }=0.0001 \\
\end{array}$ & $\begin{array}{c}\text { Adamax } \\
\text { b50 e10 } \\
\text { lr }=0.001 \\
\end{array}$ & $\begin{array}{c}\text { Adamax } \\
\text { b50 e10 } \\
\text { lr=0.01 } \\
\end{array}$ & $\begin{array}{c}\text { Adamax } \\
\text { b50 e10 } \\
\text { lr }=0.1 \\
\end{array}$ & $\begin{array}{c}\text { Adamax } \\
\text { b50 e10 } \\
\text { lr }=0.2 \\
\end{array}$ & \begin{tabular}{|c|} 
Adagrad \\
b10 e10 \\
lr $=0.0001$ \\
\end{tabular} & $\begin{array}{c}\text { Adagrad } \\
\text { b10 e10 } \\
\text { lr }=0.001 \\
\end{array}$ & $\begin{array}{c}\text { Adagrad } \\
\text { b10 e10 } \\
\text { lr=0.01 } \\
\end{array}$ \\
\hline AUC & 0.9309 & 0.9449 & 0.9422 & 0.9392 & 0.9346 & 0.7315 & 0.9271 & 0.9449 \\
\hline F1 Score & 0.4986 & 0.5897 & 0.5686 & 0.5977 & 0.6351 & 0.1149 & 0.4836 & 0.5950 \\
\hline \multirow[t]{2}{*}{ Accuracy } & 0.9206 & 0.9165 & 0.9153 & 0.9139 & 0.9116 & 0.9101 & 0.9156 & 0.9195 \\
\hline & $\begin{array}{c}\text { Adagrad } \\
\text { b10 e10 } \\
\text { lr }=0.1\end{array}$ & $\begin{array}{c}\text { Adagrad } \\
\text { b10 e10 } \\
\text { lr }=0.2\end{array}$ & \begin{tabular}{|c|} 
Adam b50 \\
e10 \\
Ir $=0.0001$ \\
\end{tabular} & $\begin{array}{c}\text { Adam } \\
\text { b50 e10 } \\
\text { Ir }=0.001\end{array}$ & $\begin{array}{c}\text { Adam } \\
\text { b50 e10 } \\
\text { lr=0.01 }\end{array}$ & $\begin{array}{l}\text { Adam b50 } \\
\text { e10 lr=0.1 }\end{array}$ & $\begin{array}{l}\text { Adam b50 } \\
\text { e10 lr }=0.2\end{array}$ & \\
\hline AUC & 0.9421 & 0.9386 & 0.9390 & 0.9436 & 0.9392 & 0.8707 & 0.4989 & \\
\hline F1 Score & 0.5765 & 0.5911 & 0.5592 & 0.5852 & 0.6067 & 0.0399 & nan & \\
\hline Accuracy & 0.9172 & 0.8878 & 0.9176 & 0.8831 & 0.9188 & 0.8884 & 0.9164 & \\
\hline
\end{tabular}

Adamax and Adagrad become flat starting with the learning rate equal to 0.001 staying flat for learning rates up to 0.2. Adam proved to be more sensitive on the learning rate variation. For the next optimizations the learning rate $1 r=0.001$ is kept as unique learning rate over all other optimizations.

Table 4. Weights initialization optimization

\begin{tabular}{|c|c|c|c||c|c|}
\cline { 2 - 6 } \multicolumn{1}{c|}{} & $\begin{array}{c}\text { In Adagrad } \\
\text { b10 e10 }\end{array}$ & $\begin{array}{c}\text { In Adamax } \\
\text { b50 e10 }\end{array}$ & $\begin{array}{c}\text { Iu Adagrad } \\
\text { b10 e10 }\end{array}$ & $\begin{array}{c}\text { Iu Adamax b50 } \\
\text { e10 }\end{array}$ & $\begin{array}{c}\text { One Adagrad } \\
\text { b10 e10 }\end{array}$ \\
\hline AUC & 0.9318 & 0.9438 & 0.9222 & 0.9453 & 0.7323 \\
\hline F1 Score & 0.4783 & 0.5909 & 0.4691 & 0.5775 & 0.3606 \\
\hline Accuracy & 0.9094 & 0.9205 & 0.9082 & 0.9183 & 0.9023 \\
\hline & $\begin{array}{c}\text { One Adamax } \\
\text { b50 e10 }\end{array}$ & $\begin{array}{c}\text { xn Adagrad } \\
\text { b10 e10 }\end{array}$ & $\begin{array}{c}\text { Xn Adamax } \\
\text { b50 e10 }\end{array}$ & $\begin{array}{c}\text { xu Adagrad } \\
\text { b10 e10 }\end{array}$ & $\begin{array}{c}\text { xu Adamax b50 } \\
\text { e10 }\end{array}$ \\
\hline AUC & 0.8077 & 0.9313 & 0.9448 & 0.9298 & 0.9456 \\
\hline F1 Score & 0.3532 & 0.4969 & 0.6050 & 0.4832 & 0.5784 \\
\hline Accuracy & 0.9026 & 0.9122 & 0.9217 & 0.9104 & 0.9177 \\
\hline
\end{tabular}

Where $\ln =$ LeCun normal, $\mathrm{lu}=$ Lecun uniform, $\mathrm{xn}=$ Xavier Normal, $\mathrm{xu}=$ Xavier Uniform.

Out of all five possible initialization strategies the one consisting of initial weights equal to 1 (One Adagrad and One Adamax) proved to be the worst. The difference between this one and the other four being around $10 \%$ less for all metrics considered. For F1 Score the difference it is even bigger going up to $40 \%$.

Table 5. Activation Function Optimization

\begin{tabular}{|c|c|c|c|c|c|c|c|c|}
\hline & $\begin{array}{c}\text { relu } \\
\text { xn }\end{array}$ & $\begin{array}{c}\text { relu } \\
\text { ln }\end{array}$ & $\begin{array}{c}\text { relu } \\
\mathbf{x u} \\
\end{array}$ & $\begin{array}{c}\text { relu } \\
\text { lu }\end{array}$ & linear xn & linear In & linear xu & linear lu \\
\hline $\mathbf{A U C}$ & 0.9448 & 0.9438 & 0.9456 & 0.9453 & 0.9349 & 0.9351 & 0.9349 & 0.9351 \\
\hline F1 Score & 0.6050 & 0.5909 & 0.5784 & 0.5775 & 0.5243 & 0.5259 & 0.5259 & 0.5247 \\
\hline \multirow[t]{2}{*}{ Accuracy } & 0.9217 & 0.9205 & 0.9177 & 0.9183 & 0.9121 & 0.9122 & 0.9122 & 0.9122 \\
\hline & sigmoid xn & sigmoid ln & sigmoid xu & sigmoid lu & $\begin{array}{c}\tanh \\
\text { xn } \\
\end{array}$ & $\begin{array}{c}\tanh \\
\ln \\
\end{array}$ & $\begin{array}{c}\tanh \\
\mathrm{xu} \\
\end{array}$ & $\begin{array}{c}\tanh \\
\text { lu } \\
\end{array}$ \\
\hline AUC & 0.9423 & 0.9429 & 0.9425 & 0.9428 & 0.9448 & 0.9433 & 0.9430 & 0.9435 \\
\hline F1 Score & 0.5805 & 0.5858 & 0.5898 & 0.5942 & 0.6019 & 0.5860 & 0.6012 & 0.5977 \\
\hline Accuracy & 0.9181 & 0.9192 & 0.9193 & 0.9202 & 0.9204 & 0.9182 & 0.9211 & 0.9198 \\
\hline
\end{tabular}


Only the activation function of the hidden layer is part of this optimization. After the weighs initialization optimization was performed $\ln , \mathrm{lu}, \mathrm{xn}$ and $\mathrm{xu}$ weights initialization strategies were selected to be part of the activation function optimization step due to the metrics closeness. Linear activation function it is outperformed by the other three activation functions. Even both LeCun normal and uniform initialization strategies are scoring very good in terms of AUC, the other metrics have lower values than Xavier strategies. Considering this, relu xn, relu xu and tanh $\mathrm{xn}$ are selected to be part of the next optimization.

Table 6. Number of neurons on the hidden layer optimization

\begin{tabular}{|c|c|c|c|c|}
\hline & $\begin{array}{l}\text { relu xn } \\
h(2 n-1) \\
\end{array}$ & $\begin{array}{c}\text { relu xn } \\
h(2 n+1) \\
\end{array}$ & $\begin{array}{c}\text { relu xn } \\
h(4 n-2) /(n-3) \\
\end{array}$ & $\begin{array}{c}\text { relu xn } \\
h(6 n+2) /(n-3) \\
\end{array}$ \\
\hline Neurons & h121 & h123 & h4 & h6 \\
\hline $\mathbf{A U C}$ & 0.9452 & 0.9448 & 0.9361 & 0.9380 \\
\hline F1 Score & 0.6075 & 0.6050 & 0.5656 & 0.5967 \\
\hline \multirow[t]{2}{*}{ Accuracy } & 0.9222 & 0.9217 & 0.9151 & 0.9193 \\
\hline & $\begin{array}{c}\text { relu xu } \\
\mathrm{h}(\mathrm{n} / 2-1)\end{array}$ & $\begin{array}{l}\tanh x u \\
h(2 n-1) \\
\end{array}$ & $\begin{array}{c}\text { relu xu } \\
h(4 n-2) /(n-3)\end{array}$ & $\begin{array}{c}\text { relu xu } \\
h(6 n+2) /(n-3)\end{array}$ \\
\hline Neurons & h29 & h121 & h4 & h6 \\
\hline $\mathbf{A U C}$ & 0.9404 & 0.9442 & 0.9357 & 0.9343 \\
\hline F1 Score & 0.5769 & 0.5974 & 0.5192 & 0.5790 \\
\hline \multirow[t]{2}{*}{ Accuracy } & 0.9165 & 0.9205 & 0.9134 & 0.9137 \\
\hline & relu xu h(2n-1) & relu xu $h(2 n+1)$ & $\tanh x u h(4 n-2) /(n-3)$ & $\tanh \times u h(2 n+1)$ \\
\hline Neurons & h121 & h123 & h4 & h123 \\
\hline AUC & 0.9462 & 0.9456 & 0.9413 & 0.9430 \\
\hline F1 Score & 0.6037 & 0.5784 & 0.6014 & 0.6012 \\
\hline \multirow[t]{2}{*}{ Accuracy } & 0.9211 & 0.9177 & 0.9199 & 0.9211 \\
\hline & $\tanh \times u h(n / 2-1)$ & relu xn $h(n / 2-1)$ & $\tanh x u h(6 n+2) /(n-3)$ & \\
\hline Neurons & h29 & h29 & h6 & \\
\hline AUC & 0.9432 & 0.9403 & 0.9427 & \\
\hline F1 Score & 0.6072 & 0.5851 & 0.5839 & \\
\hline Accuracy & 0.9209 & 0.9181 & 0.9166 & \\
\hline
\end{tabular}

Where $\mathrm{h}$ represents the hidden layer, $\mathrm{n}$ the number of input variables and inside the brackets the formula for number of neurons on the hidden layer. The best results are provided by the networks with the neurons on the hidden layer var varying around $(2 n+1)$ and $(2 n-1)$.

Table 7. Number of layers optimization

\begin{tabular}{|c|c|c|c|c|c|}
\hline & $\begin{array}{c}\text { relu xn } \\
\mathbf{h}(\mathbf{2 n - 1})\end{array}$ & $\begin{array}{c}\text { relu xn h(n/2- } \\
\mathbf{1})\end{array}$ & $\begin{array}{c}\text { relu xu } \\
\mathbf{h}(\mathbf{2 n - 1})\end{array}$ & $\begin{array}{c}\text { relu xu } \\
\mathbf{h}(\mathbf{4 n - 2}) /(\mathbf{n}-3)\end{array}$ & $\begin{array}{c}\text { relu xu } \\
\mathbf{h}(\mathbf{6 n + 2}) /(\mathbf{n}-3)\end{array}$ \\
\hline Neurons & $\mathrm{h} 121, \mathrm{hh} 241$ & $\mathrm{~h} 29, \mathrm{hh} 13$ & $\mathrm{~h} 121, \mathrm{hh} 241$ & $\mathrm{~h} 4, \mathrm{hh} 14$ & $\mathrm{~h} 6, \mathrm{hh} 12$ \\
\hline F1 Score & 0.5769 & 0.5901 & 0.5919 & 0.5718 & 0.5446 \\
\hline Accuracy & 0.9172 & 0.9172 & 0.9192 & 0.9087 & 0.9101 \\
\hline AUC & 0.9435 & 0.9397 & 0.9432 & 0.9323 & 0.9332 \\
\hline & $\begin{array}{c}\text { relu xu } \\
\mathbf{h}(\mathbf{2 n + 1})\end{array}$ & $\begin{array}{c}\text { relu xu h(n/2- } \\
\mathbf{1})\end{array}$ & $\begin{array}{c}\tanh \mathbf{x u} \\
\mathbf{h}(\mathbf{n} / \mathbf{2 - 1})\end{array}$ & $\begin{array}{c}\mathbf{t a n h} \mathbf{x u} \\
\mathbf{h}(\mathbf{4 n - 2}) /(\mathbf{n}-3)\end{array}$ & $\begin{array}{c}\mathbf{t a n h} \mathbf{x u} \\
\mathbf{h}(\mathbf{6 n + 2}) /(\mathbf{n}-3)\end{array}$ \\
\hline
\end{tabular}


www.conferenceie.ase.ro

\begin{tabular}{|c|c|c|c|c|c|}
\hline Neurons & $\mathrm{h} 123, \mathrm{hh} 247$ & $\mathrm{~h} 29, \mathrm{hh} 13$ & $\mathrm{~h} 29, \mathrm{hh} 13$ & $\mathrm{~h} 4, \mathrm{hh} 14$ & $\mathrm{~h} 6, \mathrm{hh} 12$ \\
\hline F1 Score & 0.5869 & 0.6019 & 0.5924 & 0.6052 & 0.6033 \\
\hline Accuracy & 0.9187 & 0.9149 & 0.9183 & 0.9173 & 0.9184 \\
\hline AUC & 0.9436 & 0.9398 & 0.9422 & 0.9417 & 0.9421 \\
\hline & $\begin{array}{c}\text { relu xn } \\
\mathbf{h}(\mathbf{2 n + 1})\end{array}$ & $\begin{array}{c}\text { tanh } \mathbf{x u} \mathbf{h}(\mathbf{2 n}- \\
\mathbf{1})\end{array}$ & $\begin{array}{c}\mathbf{t a n h} \mathbf{x u} \\
\mathbf{h}(\mathbf{2 n + 1})\end{array}$ & $\begin{array}{c}\text { relu xn } \\
\mathbf{h}(\mathbf{4 n - 2}) /(\mathbf{n}-\mathbf{3})\end{array}$ & $\begin{array}{c}\text { relu xn } \\
\mathbf{h}(\mathbf{6 n + 2}) /(\mathbf{n}-\mathbf{3})\end{array}$ \\
\hline Neurons & $\mathrm{h} 123, \mathrm{hh} 247$ & $\mathrm{~h} 121, \mathrm{hh} 241$ & $\mathrm{~h} 123, \mathrm{hh} 247$ & $\mathrm{~h} 4, \mathrm{hh} 14$ & $\mathrm{~h} 6, \mathrm{hh} 12$ \\
\hline F1 Score & 0.5925 & 0.5896 & 0.6002 & 0.6055 & 0.5661 \\
\hline Accuracy & 0.9195 & 0.9160 & 0.9192 & 0.9081 & 0.9124 \\
\hline AUC & 0.9438 & 0.9424 & 0.9419 & 0.9323 & 0.9367 \\
\hline
\end{tabular}

Notation convention made for a single hidden layer it is kept for the second layer optimization and additionally hh stand for second hidden layer. The number of neurons of the second hidden layer is calculated considering the first hidden layer input for it. Adding the second hidden layer is not adding any improvement on the metrics, but it is adding computational power and time for the training. For the studied use case the best architecture it is a Multi-layer perceptron with one hidden layer consisting of 121 neurons, Relu activation function for the hidden layer, Sigmoid for output layer and Adamax optimizer. Weights initialization it is made using Xavier uniform strategy.

Table 8. Comparison between baseline and final selected model

\begin{tabular}{|c|c|c|}
\hline & bable 8. Comparison between baseline and final selected model \\
\hline Neurons & 123 & relu adamax b50 e10 xu h(2n-1) model \\
\hline AUC & 0.9377 & 121 \\
\hline F1 Score & 0.5174 & 0.9462 \\
\hline Accuracy & 0.9124 & 0.6037 \\
\hline
\end{tabular}

\section{Conclusion}

Technological advance it is creating the premise for gathering more and more. Setting the right skill set in terms of data mining and subject matter is essential to bring value into and to remain competitive. This study fills the gap in the data driven decision systems for marketing campaigns presenting an end-to-end approach for increasing effectiveness. The deep dive made on both theoretical and experimental aspects of hyperparameter tuning give right image over how much the right setting can make the difference between benchmark performance and poor performance. Weights Initialization and Activation function proved to impact most the performance of the neural network. The negative impact on the selected hyperparamters can go up to $40 \%$. The best hyperparameters configuration improved some of the existing work done on the same data set. However, the baseline model proved to be competitive even without optimization and this fact is only highlighting again one of the premises of this research, Artificial Neural Networks can act like universal approximators being able to find patters in the data for benchmark results.

\section{References}

[1] N. Hänninen, H. Karjaluoto, "The effect of marketing communication on business relationship loyalty", Marketing Intelligence \& Planning, vol. 35, no. 4, pp. 458-472, June 2017. 
[2] C. Matt, T. Hess, A. Benlian, "Digital Transformation Strategies", Business \& Information Systems Engineering, vol. 57, no. 5, pp. 339-343, September 2015.

[3] C. Lamberton, A. T. Stephen, "A Thematic Exploration of Digital, Social Media, and Mobile Marketing Research's Evolution from 2000 to 2015 and an Agenda for Future Research", Journal of Marketing, vol. 80, pp. 146-172, June 2016.

[4] K. Janssens, N. Nijsten, R. Van Goolen, "Spam and Marketing Communications", Procedia Economics and Finance, vol. 12, pp.265-272, December 2014.

[5] N. S. Ahmad, R. Musa, M. H. M. Harun, "The Impact of Social Media Content Marketing (SMCM) towards Brand Health", Procedia Economics and Finance, vol. 37, pp. 331-336, December 2016.

[6] J. Wei, S. Lin, H. Wu, "A review of the application of RFM model", African journal of business management, vol. 4, pp.4199-4206, January 2010.

[7] A. Zakaryazad, E. Duman, "A profit-driven Artificial Neural Network (ANN) with applications to fraud detection and direct marketing", Neurocomputing, 175 (2016), pp. 121-131.

[8] FabriceTalla Nobibon, Roel Leus, Frits C.R.Spieksma, "Optimization models for targeted offers in direct marketing: Exact and heuristic algorithms", European Journal of Operational Research, vol. 210, no.3, pp. 670-683, May 2011.

[9] W. Reinartz, J.S. Thomas, V. Kumar, "Balancing acquisition and retention resources to maximize customer profitability", Journal of Marketing, vol. 69, pp. 63-79, January 2005.

[10] Y. Deliana, I. A. Rum, "Understanding consumer loyalty using neural network", Polish Journal of Management Studies, vol. 116, no. 2, pp. 51-61, December 2017.

[11] A. Oztekin, "Creating a marketing strategy in healthcare industry: a holistic data analytic approach", Annals of Operations Research, vol. 270, pp. 361-382, May 2017.

[12] W. Wang, M. M. Santos Silva, L. Moutinho, "Modelling Consumer Responses to Advertising Slogans through Artificial Neural Networks", International Journal of Business and Conomics, vol. 15.

[13] H. Elsalamony, "Bank Direct Marketing Analysis of Data Mining Techniques", International Journal of Computer Applications, vol. 85, no. 7, pp. 12-22, December 2013.

[14] M. Scherer, J. Smolag, A. E. Gaweda, "Predicting Success of Bank Direct Marketing by Neuro-fuzzy Systems", Artificial Intelligence and Soft Computing: 15th International Conference, ICAISC 2016, Proceedings, Part II, pp. 570-576, June 2016.

[15] W. S. McCulloch, W. Pitts, "A logical calculus of the ideas immanent in nervous activity", The bulletin of mathematical biophisics, vol. 5, no. 5, pp 115-133, December 1943

[16] M. Li, T. Zhang, Y. Chen, A. J. Smola, "Efficient mini-batch training for stochastic optimization", Proceedings of the ACM SIGKDD International Conference on Knowledge Discovery and Data Mining, pp. 661-670, August 2014.

[17] I. Goodfellow, Y. Bengio, A. Courville, Deep Learning (Adaptive Computation Machine Learning series), The MIT Press, pp. 82-93, 2016.

[18] F. Zou, L. Shen, "On the Convergence of Weighted AdaGrad with Momentum for Training Deep Neural Networks", August 2018.

[19] A. A. Lydia, F. S. Francis, "Adagrad-An Optimizer for Stochastic Gradient Descent", International Journal of Information and Computing Science, vol. 6, no. 5, pp. 566-568, May 2019.

[20] G. Hinton, N. Srivastava, K. Swersky, "Lecture 6a, Overview of mini-batch gradient descent",

Internet:https://www.cs.toronto.edu/ tijmen/csc321/slides/lecture_slides_lec6.pdf, March 29, 2020. 
[21] D. Kingma, J. Ba, "Adam: A Method for Stochastic Optimization",International Conference on Learning Representations,pp.1-15, 2014.

[22] Y.A. LeCun, L.Bottou, G.B. Orr, KR Muller, "Efficient BackProp", Neural Networks: Tricks of the Trade. Lecture Notes in Computer Science, vol 7700, pp. 9-48, 2012

[23] X. Glorot, Y. Bengio, "Understanding the difficulty of training deep feedforward neural networks", Journal of Machine Learning Research, vol. 9, pp. 249-256, January 2010.

[24] D. Graupe,Principles of Artificial Neural Networks, World Scientific, pp.19-21, 2016

[25] A. Krizhevsky, I. Sutskever, and G. E. Hinton, "ImageNet classification with deep convolutional neural networks," Communications of the ACM, vol. 60, no. 6, pp. 84-90, May 2017.

[26] D. Hunter, H. Yu, M. S. Pukish, III, J. Kolbusz, B. M. Wilamowski, "Selection of Proper Neural Network Sizes and Architectures-A Comparative Study, IEEE Transactions on Industrial Informatics, VOL. 8, NO. 2, MAY 2012.

[27] M.Madhiarasan, S.N Deepa,"Comparative analysis on hidden neurons estimation in multi layer perceptron neural networks for wind speed forecasting", Artificial Intelligence Review, vol. 48, pp. 449-471, 2017.

[28] p. Gaurang, A. Ganatra, Y. P. Kosta, D. Panchal, "Behavior Analysis of Multipayer Perceptrons with Multiple Hidden Neurons and Hidden Layers", International Journal of Computer Theory and Engineering, Vol. 3, No.2, April 2011.

[29] S. Moro, P. Cortez, P. Rita, "A data-driven approach to predict the success of bank telemarketing”, Decision Support Systems, vol. 62, pp. 22-31, March 2014.

[30] M. Mehryar, A. Rostamizadeh, A. Talwalkar, Foundations of Machine Learning, The MIT Press, pp. 6-7, 2018.

[31] K. Potdar, T. S. Pardawala, C.D. Pai, "A Comparative Study of Categorical Variable Encoding Techniques for Neural Network Classifiers", International Journal of Computer Applications, vol. 175, no. 4, October 2017.

[32] F. Provost, T. Fawcett, Data Science for Business What you need to know about data mining and data-analytic thinking, O’Relly, pp. 209-222, 2013. 